\title{
Evaluación y restauración ecológica "Lisan Wasi" comunidad San Pedro, parroquia Tarqui, Cantón Pastaza
}

\section{Ecological evaluation and restoration "Lisan Wasi”" San Pedro community, Tarqui parish, Pastaza canton}

Jessy Paulina Guerrero Rubio ${ }^{1 *}$, Angélica María Tasambay Salazar ${ }^{1}$, Fernando Cofre Santos $^{1}$, Cristian Santiago Jácome Segovia ${ }^{1}$, Carlos Raúl Valverde Lara ${ }^{1}$, Yusimy Jiménez Rojas ${ }^{1}$.

\author{
${ }^{1}$ Universidad Estatal Amazónica, Puyo, Ecuador \\ *Correspondencia: jpguerrero@uea.edu.ec
}

Rec.: 19.05.2019. Acept.: 03.01.2020 Publicado el 30 de junio de 2020

\begin{abstract}
Resumen
La vegetación es un recurso natural clave para el equilibrio del ecosistema, es necesario disponer de información cuantitativa sobre sus características y distribución. Esta investigación tuvo como objetivo, evaluar áreas perturbadas por actividades antrópicas y permitió establecer un plan de restauración ecológica con especies nativas del bosque secundario de la Amazonia ecuatoriana, en la Comunidad de San Pedro "Lisan Wasi", Parroquia Tarquí, Cantón Pastaza, se realizó un inventario florístico, se determinó los índices de valor de importancia ecológica e importancia ecológica ampliada. A demás se obtuvieron el índice de riqueza específica de Menhinick, y Margalef, de equidad de Shannon - Weaner, de dominancia de Simpson para conocer la diversidad de la vegetación. Así como el Índice de Jaccard, Sorensen y el conglomerado jerárquico. Se evaluó el grado de perturbación del área mediante indicadores de cambios en el ecosistema, como resultado se identificaron un total 33 familias, y 74 especies pertenecientes principalmente al estrato arbóreo, arbustivo y herbáceo. Las especies representativas fueron Bromeliaceae Catopsis, Cedrela odorata, Cecropia peltata, Persea americana, Schizolobium parahybum. Las familias más representativas, en este orden: Fabaceae, Aracaceae, Poaceae, Rutaceae y Melastomataceae. Los valores de los índices mostraron que existe una gran cantidad de especies, que la comunidad posee especies dominantes y por lo tanto la diversidad es mayor; no existió una formación equitativa y todas las especies no son abundantes. El grado de perturbación identificado fue por alteración a la composición de especies y alteración a la estructura, del diagnóstico realizado permitió aplicar las técnicas de restauración ecológica para el mejoramiento del área de estudio, al realizar el trasplante con especies nativas en un área de 0.24 ha, en las áreas afectadas.
\end{abstract}

Palabras claves: Restauración, ecosistema, plántulas, perturbación, trasplante

\begin{abstract}
Vegetation is a key natural resource for ecosystem balance, it is necessary to have quantitative information about its characteristics and distribution. This research aimed to evaluate areas disturbed by anthropic activities and allowed to establish an ecological restoration plan with native species of the secondary forest of the Ecuadorian Amazon, in the Community of San Pedro "Lisan Wasi", Parish Tarquí, Canton of Pastaza, was carried out a floristic inventory, the indices of value of ecological importance and extended ecological importance were determined. In addition, the specific wealth index of Menhinick, and Margalef, of Shannon-Weaner equity, Simpson's dominance to know the diversity of the vegetation was obtaided. As well as the Jaccard, Sorensen Indice and the hierarchical conglomerate. The degree of disturbance of the area was evaluated through indicators of changes in the ecosystem, as a result a total of 33 families were identified, and 74 species belonging mainly to the tree, shrub and herbaceous stratum. Representative species were Bromeliaceae catopsis, Cedrela odorata, Cecropia peltata, Persea americana, Schizolobium parahybum. The most representative families, in this order: Fabaceae, Aracaceae, Poaceae, Rutaceae and Melastomataceae. Index values showed that there is a large number of species that the community has dominant species and therefore the diversity is greater; there was no equitable formation and all species are not abundant. The degree of disturbance identified was due to alteration to the composition of species and alteration to the structure, the diagnosis allowed to apply the techniques of ecological restoration for the improvement of the study area, through the transplantation with native species in an area of 0.24 ha, in the affected areas.
\end{abstract}

Keywords: Restoration, ecosystem, seedlings, disturbance, transplant 


\section{Introducción}

$\mathrm{E}$ 1 Ecuador, es un país mega diverso que poseen una gran cantidad de climas y microclimas está conformado por 25 de las 35 Zonas de vida según la clasificación de Holdridge, por lo que se puede mencionar el encuadre biogeográfico capaz de encerrar en espacios muy reducidos un ecosistema que posee el mayor número de individuos en una porción de terreno. Es importante mencionar que los bosques húmedos por tener en $1 \mathrm{Km}^{2}$ aproximadamente, 12500 especies de plantas de diferente familia (Mera, 2018).

La conservación comprende la protección de las estructuras, composición y funcionamiento de los niveles de organización de un ecosistema, que constituyen la biodiversidad. Su protección es una problemática compleja, que requiere de un entendimiento profundo de la interrelación ambiente-sociedad en espacios geográficamente concretos. La variedad y cantidad de tipos de vegetación son indicadores relevantes en el análisis de la biodiversidad de un ecosistema para su conservación (Luebert y Becerra, 1998). Los inventarios describen la funcionalidad de la estructura vegetal para su aplicación en el uso y manejo de la misma (Álvarez et al., 2006). La caracterización de sus propiedades fisiológicas y morfológicas, permite el reconocimiento de la complejidad estructural presente, mientras que su representación mediante fórmulas resume la información en un solo valor. También es posible realizar comparaciones entre la diversidad de distintos hábitats o la diversidad de un mismo hábitat a través del tiempo y espacio. Los índices cuantitativos muestran la relevancia de su conservación en áreas (Suárez y Vischi, 1997).

La Amazonia ecuatoriana, se caracteriza por tener una exuberante vegetación, misma que provee beneficios ambientales como: purificar el aire, evitar la erosión de los suelos, mejorar la calidad del paisaje y reducir la contaminación litológica. De igual manera está formada por distintas poblaciones étnicas de comunidades ancestrales que se encuentran acentuadas en los ecosistemas endémicos de la región. Históricamente los bosques han sido intervenidos para la extracción de recursos naturales lo que ha provocado una afectación en su estructura vegetal, funcional y dinámica. La deforestación intensiva es uno de los factores principales que afectan a la región Amazónica dando como resultado un cambio en el suelo y pérdida de la fauna y flora endémica de la zona.

En la actualidad la pérdida acelerada de la biodiversidad en el Emprendimiento Turístico Lisan Wasi, comunidad de San Pedro, por las actividades antropogénicas del hombre ha conllevado a la destrucción de los recursos naturales. Con los antecedentes mencionados la presente investigación se enfoca en evaluar y caracterizar las zonas degradadas en Lisan Wasi, así como también identificar las especies vegetales en la zona y las formas de distribución según las variables ambientales.

La presente investigación contribuirá para la aplicación de mecanismos requeridos para la restauración de áreas afectadas en el área intervenida, servirá como guía para futuras investigaciones de conservación y desarrollo turístico, que permitirá conocer las especies nativas del sector y la riqueza vegetal, los cuales garantizarán la importancia de la conservación de las condiciones ecológicas.

\section{Materiales y métodos}

T a presente investigación se realizó en 5 transeptos - permanentes de muestreo, con una área total 0.24 ha. en las proximidades del río Puyo, en el Emprendimiento Turístico Lisan Wasi, ubicado a $21 \mathrm{~km}$ de la ciudad de Puyo en la comunidad de San Pedro, Parroquia Tarqui, Cantón y Provincia Pastaza, (Figura 1). Los transeptos se encuentran en un bosque secundario en regeneración natural, Clima superhúmedo sin déficit de agua, Mesotérmico templado cálido, al este de la cordillera de oriental entre los 600 a $700 \mathrm{msnm}$, en la amazonia Ecuatoriana.

\section{Inventario florístico}

Se realizó un inventario florístico de las estructuras vegetales, con diámetros mayores e iguales a $10 \mathrm{~cm}(\mathrm{~d} 1.30 \geq 10 \mathrm{~cm})$, los ejemplares botánicos se colectaron en las áreas de estudio, utilizando siempre para ello guías del lugar, quienes nos indicaron los lugares más conservados, teniendo como prioridad que dichos ejemplares presentaran flor $\mathrm{y} / \mathrm{o}$ fruto. Se procuró colectar duplicados por número, las colectas se realizaron mediante el conteo físico por especie de acuerdo a la metodología de Lot y Chiang (1986). Se realizó la identificación botánica preliminar en el campo y se confirmó con la literatura apropiada: Manual de Botánica Sistemática, Etnobotánica y Métodos de Estudio en el Ecuador (Cerón, 1993), Libro Rojo de las Plantas Endémicas del Ecuador (León et al.,2012), empleado para la clasificación de las angiospermas además fueron constatadas en el herbario Ecuatoriano Amazónico (ECAAMZ).

\section{Parámetros ecológicos} Valor de importancia ecológica

El índice de valor de importancia calculada mediante la ecuación (1), define cuáles de las especies presentes 


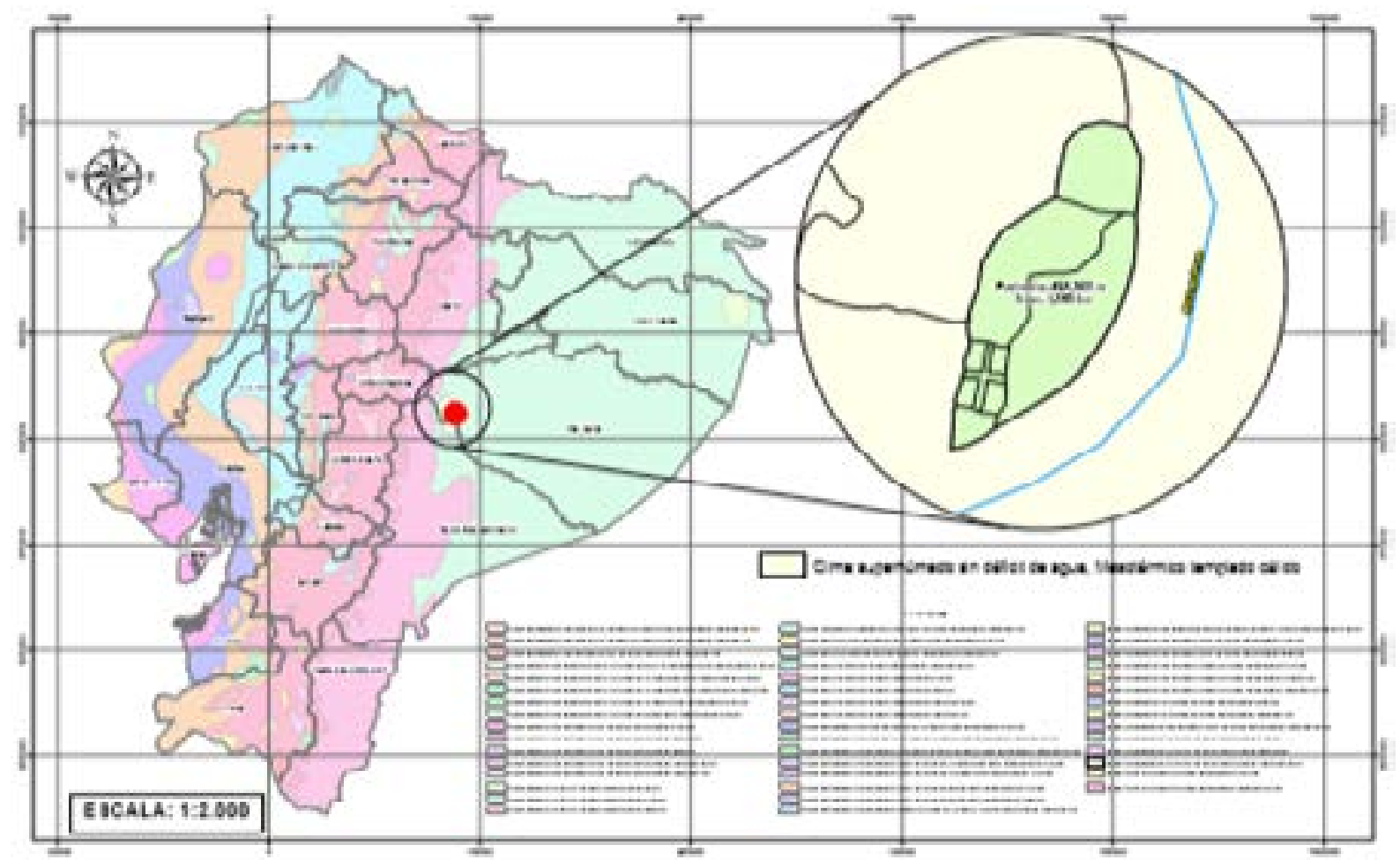

Figura1. Mapa con la ubicación geográfica del área de estudio

contribuyen en el carácter y estructura de un ecosistema metodología propuesta por (Cottam y Curtis, 1956). Este valor se obtiene mediante la sumatoria de la frecuencia relativa (2), la densidad relativa (3) y la dominancia relativa (4)

$$
\mathrm{IVIE}=(\mathrm{AR}+\mathrm{FR}+\mathrm{DR}) / 3
$$

Donde:

\section{$\mathbf{A R}=$ Abundancia relativa}

$\mathrm{AR}=\underline{\text { Núm. de árboles por sp }} \times 100$

Núm. de árboles totales

\section{$\mathrm{FR}=$ Frecuencia relativa}

$\mathrm{FR}=$ Frecuencia de la sp $\times 100$

Frecuencia de todas las spp

\section{$\mathrm{DR}=$ Dominancia relativa}

$\mathrm{DR}=\underline{\text { área basal de cada especie }} \times 100$ área basal de todas las spp

Índice de valor de importancia ecológica ampliado (IVIEA)

Con los valores de RNRi y el IVIE se determinó el índice de valor de importancia ecológica ampliado, para determinar la perpetuidad de la especie y la importancia fitosociológica de cada una de ellas, según lo propuesto por Finol (1971), mediante la ecuación (5)

$$
\text { IVIEA }=\text { IVIE }+ \text { RNR }
$$

Donde:

IVIE $=$ Índice de valor de importancia ecológica $\mathrm{RNRi}=$ Porcentaje de la proporción de cada especie regenerada

IVIE = Índice de valor de importancia ecológica $\mathrm{RNRi}=$ Porcentaje de la proporción de cada especie regenerada

\section{Diversidad alfa \\ Riqueza específica}

\section{Índice Menhinick}

El índice de diversidad de Menhinick (Dmn) (6) al igual que el índice de Margalef se basa en la relación entre el número de especies y el número total de individuos observados, que aumenta al aumentar el tamaño de la muestra.

$$
D m n=-\frac{\mathrm{S}}{\sqrt{\mathrm{N}}}
$$




\section{Índice Margalef}

El índice de Margalef(Dmg) (7) es una forma sencilla de medir la biodiversidad ya que proporciona datos de riqueza de especies de la vegetación. Mide el número de especies por número de individuos especificados o la cantidad de especies por área en una muestra (Margalef, 1969).

$$
\mathrm{Dmg}=\frac{\mathrm{S}-1}{\mathrm{LnN}} \quad \lambda=\sum \mathrm{pi}^{2}
$$

Dónde:

$\mathbf{S}=$ Número total de especies

$\mathbf{N}=$ Número total de individuos

\section{Estructura (dominancia y equidad)}

Índice de equidad de Shannon - Weaner

El índice de Shannon-Wiener $\left(\mathrm{H}^{\prime}\right)(8)$ tiene en cuenta la riqueza de especies y su abundancia. Este índice relaciona el número de especies con la proporción de individuos pertenecientes a cada una de ellas presente en la muestra. Además mide la uniformidad de la distribución de los individuos entre las especies (9). Su fórmula es:

$$
\mathbf{H}^{\prime}=\Sigma \mathrm{p}_{\mathrm{i}} \cdot \ln \mathrm{p}_{\mathrm{i}}
$$

Dónde:

$\mathbf{p}_{\mathbf{i}}=$ probabilidad de la especie $\mathbf{i}$ respecto al conjunto.

$$
p_{i}=\frac{N_{i}}{N}
$$

Dónde:

$\mathbf{N}_{\mathrm{i}}=$ Número de individuos de la especie $\mathrm{i}$

$\mathbf{N}=$ Número total de individuos de la muestra

\section{Índice de Simpson}

El índice de dominancia de Simpson (DSi) (10) considera la probabilidad que dos individuos de la población seleccionados al azar sean de la misma especie. Indica la relación existente entre riqueza o número de especies y la abundancia o número de individuos por especie. Dada por la siguiente expresión:

\section{Dónde:}

$\mathbf{P i}=$ abundancia proporcional de la especie $i$, es decir, el número de individuos de la especie i dividido entre el número total de individuos de la muestra.

\section{Diversidad beta}

La diversidad beta ha sido usada para expresar el reemplazo espacial en la identidad de las especies entre dos o más áreas y es una medida en la diferencia de la composición de especies entre dos o más ensamblajes locales o regionales (Koleff, 2005). La similitud florística entre los sitios de estudio se obtuvo mediante el índice de similitud de Jaccard y Sorensen para el análisis cualitativo de presencia - ausencia de especies, los cuales permiten contribuir al mejor entendimiento de las estructuras (Magurran, 1988; Koleff, 2005), se realizó un análisis de conglomerado jerárquico a partir de los incides de los índices de similitud de Jaccard y Sorencen, lo cual permitió la clasificación de las parcelas por su composición florística utilizando el programa Bio-diversity Professional (McAleece, 1997), según la ecuación (11) y (12)

$$
\begin{aligned}
& \text { Indicé de Jaccard }=\frac{c}{(a+b-c)} \\
& \text { Indicé de Sorensen }=\frac{2 c}{(a+b)}
\end{aligned}
$$

Dónde:

$\mathrm{a}=$ número de especies en el sitio $\mathrm{A}$

$\mathrm{b}=$ número de especies en el sitio $\mathrm{B}$

$\mathrm{c}=$ número de especies presentes en ambos sitios $\mathrm{A}$ y $\mathrm{B}$,

\section{Evaluación del grado de perturbación}

La evaluación del grado de perturbación se realizó a partir de los criterios de Matos y Ballate (2006), basado en modificaciones del método propuesto, y con adecuaciones para este trabajo respecto a la evaluación del efecto provocado por actividades antrópicas en las parcelas seleccionadas, considerando: grado de representatividad de las especies originales, grado de representatividad de los estratos originales de la vegetación, grado de cobertura vegetal y grado de modificación o perturbación. Para evaluar el grado de representatividad de los estratos originales se tuvo en cuenta la existencia de los estratos característicos en la vegetación actual (arbóreo, arbustivo, herbáceo), y la caracterización fisonómica del área en correspondencia con la composición florística representativa, así como mediciones de los estratos en relación con la altura característica que constituyen elementos de gran valor para el diagnóstico. La cobertura vegetal se evalúo a partir del análisis del porcentaje que ocupa la vegetación, mediante la comparación de las variables de conservación ideal y de conservación real en observaciones directas realizadas en el área de estudio mediante trabajo de campo y los criterios asumidos. Para la evaluación del grado de modificación del área se tuvo en cuenta los indicadores: área ocupada por caminos, extracción de recursos vegetales, ocurrencia de incendios forestales, talas, pastoreo e incidencia de vegetación secundaria e introducida. En cada uno de estos indicadores se utilizaron matrices que evalúan 


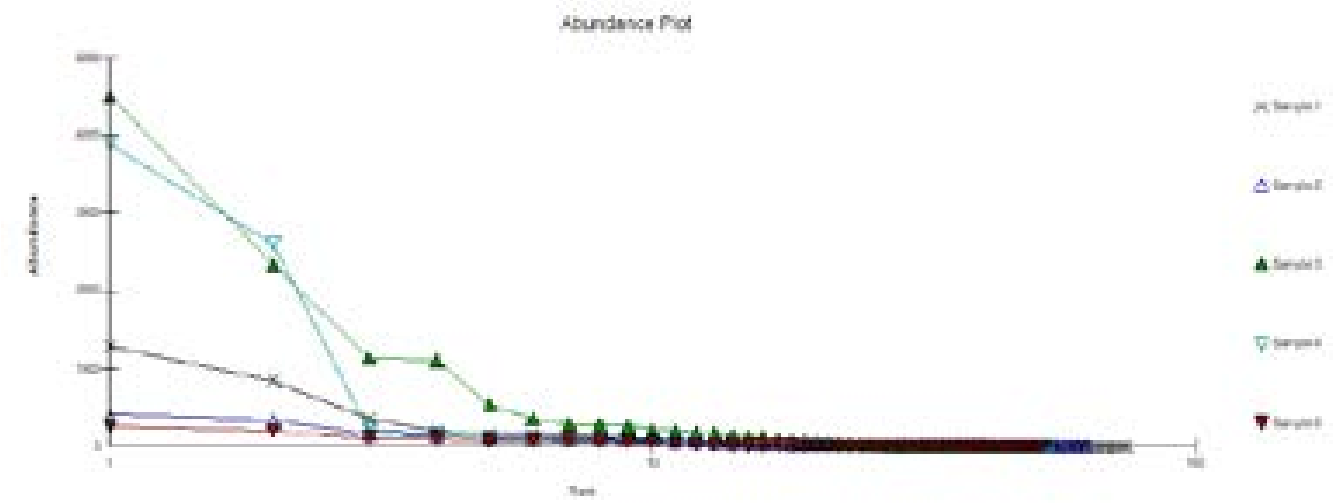

Figura 2. Curva de parcela de abundancia de las especies Bromeliaceae Catopsis, Cedrela odorata, Cecropia peltata, Persea americana, Schizolobium parahybum

cada factor modificante para transformar las variables cualitativas a cuantitativas, mediante la asignación de valores escalares de 0 hasta 3 de acuerdo al grado de perturbación del área, considerando 0 (muy alto grado de perturbación) cuando la suma de los indicadores se encuentra entre 1-4 puntos, 1 (alto grado de perturbación) entre 5-14 puntos, 2 (medio grado de perturbación) entre 15-20 puntos y 3 (bajo grado de perturbación) cuando se encuentra por encima de 20 puntos.

\section{Resultados y discusión}

\section{Composición florística.}

En el inventario florístico se identificó un total 33 familias, y 74 especies, lo que resulta un área florísticamente abundante como se muestra en la figura 2 , donde predomina las cinco especies más importantes Bromeliaceae Catopsis, Cedrela odorata, Cecropia peltata, Persea americana, Schizolobium parahybum. Las familias más representativas, en este orden: Fabaceae, Aracaceae, Poaceae, Rutaceae y Melastomataceae.

En un estudio similar Patiño et al (2015) analizaron la composición florística y estructura de un bosque siempreverde piemontano de 600 a 700 m.s.n.m en la cuenca del río Piatúa en la Provincia de Napo, en el cual identificaron 32 familias, 68 especies, las familias con mayor número de especies corresponden a Aracaceae, Fabaceae y Moraceae.

\section{Estructura de bosque}

En el cuadro 1 se muestran los parámetros estructurales del bosque secundario determinados por el IVIE, RNNi e IVIEA. Los valores del IVIE como resultado de la abundancia, frecuencia relativa $\mathrm{y}$ dominancia indicaron que Schizolobium parahybum (33.28\%) es la de mayor importancia ecológica, seguido de Drimys winteri (4.01\%), Bixa Orellana (3.73\%), Bactris gasipaes (3.49\%), Cedrela odorata (3.00\%). El menor valor fue la especie Dacryodes peruviana
$(0.36 \%)$. Las especies que presentaron mayor IVIE coinciden con las de mayor valor de RNRi. El mayor valor de IVIEA lo presentó Schizolobium parahybum (39.31\%), seguido de Drimys winteri (9.98\%), Bixa Orellana (7.00\%), Bactris gasipaes (8.56\%), Cedrela odorata $(37.31 \%)$; resultado que no difiere del IVIE dado la mayor RNRi que presentó $S$. parahybum, demostrando que es la especie que presenta mayor generación natural. Para Patiño et al (2014) determianrón las especies con mayor IVI a Iriartea deltiloidea, Nectrandra sp., Ocotea aciphylla, Otoba parvifolia. Lo que se puede determinar que al ser zonas geográficas similares en las cuales existen variación del estrato arbóreo, esto en el caso del área de estudio puede darse a la actividad antrópica y la regeneración natural de las especies que tiene a lugar considerando que es un bosque secundario.

\section{Diversidad alfa}

La diversidad alfa estimada mediante el índice de Menhinick, en las 5 parcelas de estudio resulto de una valoración medio-alta con un valor de 0.44 ; mientras que los resultados para el índice de Margalef, fue de 7.39; estos resultados reafirman que en las parcelas las especies presentan una distribución heterogénea, aunque existan diferencias en el número de individuos de cada uno de los sitios. En estudios similares en la Provincia de Napo define este tipo de ecosistema como un bosque altamente heterogéneo (Gentry, 1988). Estos resultados también contrastan con lo reportado por Samaniego et al., (2015) en el bosque siempreverde piemontano presenta una alta diversidad florística. El índice de Shannon-Wiener adquiere valores entre 0 cuando hay una sola especie y el logaritmo neperiano $(\mathrm{ln})$ de $\mathrm{S}$ cuando todas las especies están representadas por el mismo número de individuos. La determinación de este índice fue de 3.01 por lo tanto demuestra que esta comunidad es equitativa, demostrando que el bosque tiene una distribución abundante, como se muestra en la Figura 3. 
Cuadro 1. Parámetros estructurales del bosque secundario

\begin{tabular}{|c|c|c|c|}
\hline ESPECIES & IVIE & RNRi & IVIEA \\
\hline Trichilia $s p$ & 0.79 & 1.11 & 1.90 \\
\hline Erythrina crista-galli & 1.77 & 2.64 & 4.40 \\
\hline Croton lechleri & 1.41 & 2.08 & 3.49 \\
\hline Bactris gasipaes & 3.49 & 5.07 & 8.56 \\
\hline Ochroma pyramidale & 1.82 & 2.71 & 4.53 \\
\hline Pollalesta discolor & 1.65 & 2.43 & 4.08 \\
\hline Melastoma candidum & 2.04 & 2.75 & 4.79 \\
\hline Wettinia mayensis & 1.67 & 2.47 & 4.14 \\
\hline Cecropia peltata & 2.50 & 3.72 & 6.22 \\
\hline Cedrela odorata & 3.00 & 4.31 & 7.31 \\
\hline Bixa Orellana & 3.73 & 3.27 & 7.00 \\
\hline Swietenia macrophylla & 1.80 & 2.68 & 4.47 \\
\hline Drimys winteri & 4.01 & 5.97 & 9.98 \\
\hline Ficus luschnathiana & 1.88 & 2.82 & 4.70 \\
\hline Bauhinia tarapotensis & 1.40 & 2.05 & 3.45 \\
\hline Citrus $\times$ limonia & 1.92 & 2.86 & 4.78 \\
\hline Inga feuilleei & 1.66 & 2.33 & 3.99 \\
\hline Visma sp. & 1.33 & 1.99 & 3.31 \\
\hline Pourouma cecropiifolia & 1.78 & 2.58 & 4.36 \\
\hline Cojoba arbórea & 1.24 & 1.85 & 3.09 \\
\hline Cordia alliodora & 1.73 & 2.54 & 4.27 \\
\hline Protium copal & 1.33 & 1.99 & 3.31 \\
\hline Inga edulis & 2.67 & 3.97 & 6.63 \\
\hline Hevea brasiliensis & 1.47 & 1.99 & 3.45 \\
\hline Ilex guayusa & 1.25 & 1.85 & 3.10 \\
\hline Chrysophyllum cainito & 1.43 & 2.12 & 3.56 \\
\hline Schizolobium parahybum & 33.27 & 6.04 & 39.31 \\
\hline Eugenia stipitata & 1.25 & 1.71 & 2.96 \\
\hline Citrus $x$ aurantifolia & 2.28 & 2.79 & 5.06 \\
\hline Citrus $x$ paradisi & 2.43 & 3.65 & 6.08 \\
\hline Cinnamomum verum & 1.26 & 1.53 & 2.79 \\
\hline Parkia multijuga & 1.61 & 1.95 & 3.56 \\
\hline Otoba parvifolia & 1.07 & 1.60 & 2.67 \\
\hline Phytelephas aequatorialis & 0.94 & 1.28 & 2.22 \\
\hline Cecropia telenitida & 1.09 & 1.63 & 2.72 \\
\hline Mauritia flexuosa & 0.43 & 0.52 & 0.95 \\
\hline Heliocarpus americanus & 0.59 & 0.87 & 1.45 \\
\hline Guadua angustifolia & 0.77 & 1.14 & 1.91 \\
\hline Howea forsteriana & 0.50 & 0.73 & 1.22 \\
\hline Psidium guajava & 0.49 & 0.73 & 1.22 \\
\hline Cedrelinga catenaeformis & 0.41 & 0.59 & 1.00 \\
\hline Dacryodes peruviana & 0.36 & 0.52 & 0.88 \\
\hline Annona muricata & 0.40 & 0.59 & 0.99 \\
\hline
\end{tabular}

El índice de dominancia de Simpson muestra resultados entre 0 a 1 en el cual los valores cercanos a 1 explican la dominancia de las especie por sobre las demás, es así que se obtuvo como resultado 0.87 por lo tanto muestra especies dominantes, y agrupaciones con una varianza heterogénea. Si bien
Cordyline fruticosa es la especie que cuenta con mayor cantidad de individuos hay otras como Calathea lutea, Drimys winteri e Inga edulis que están representadas de la misma forma por lo cual demuestra que es una formación vegetal muy diversa. 


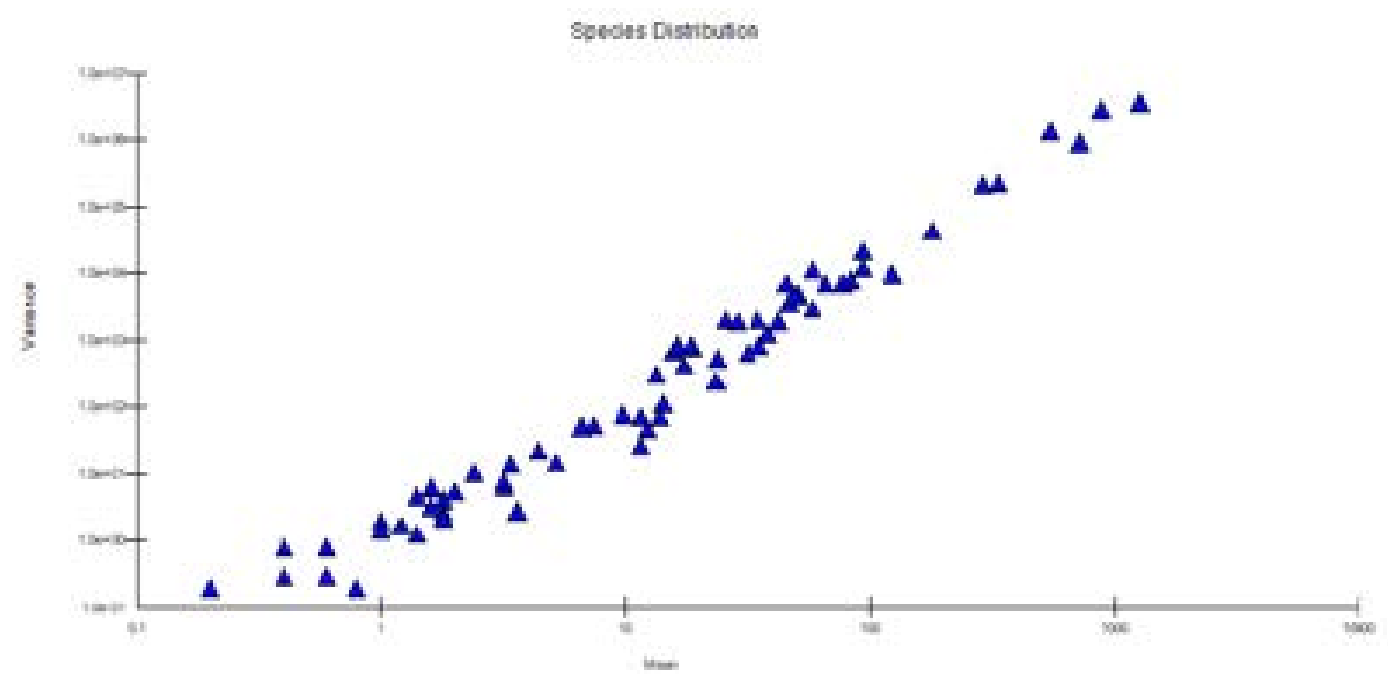

Figura 3. Distribución de especies por rango

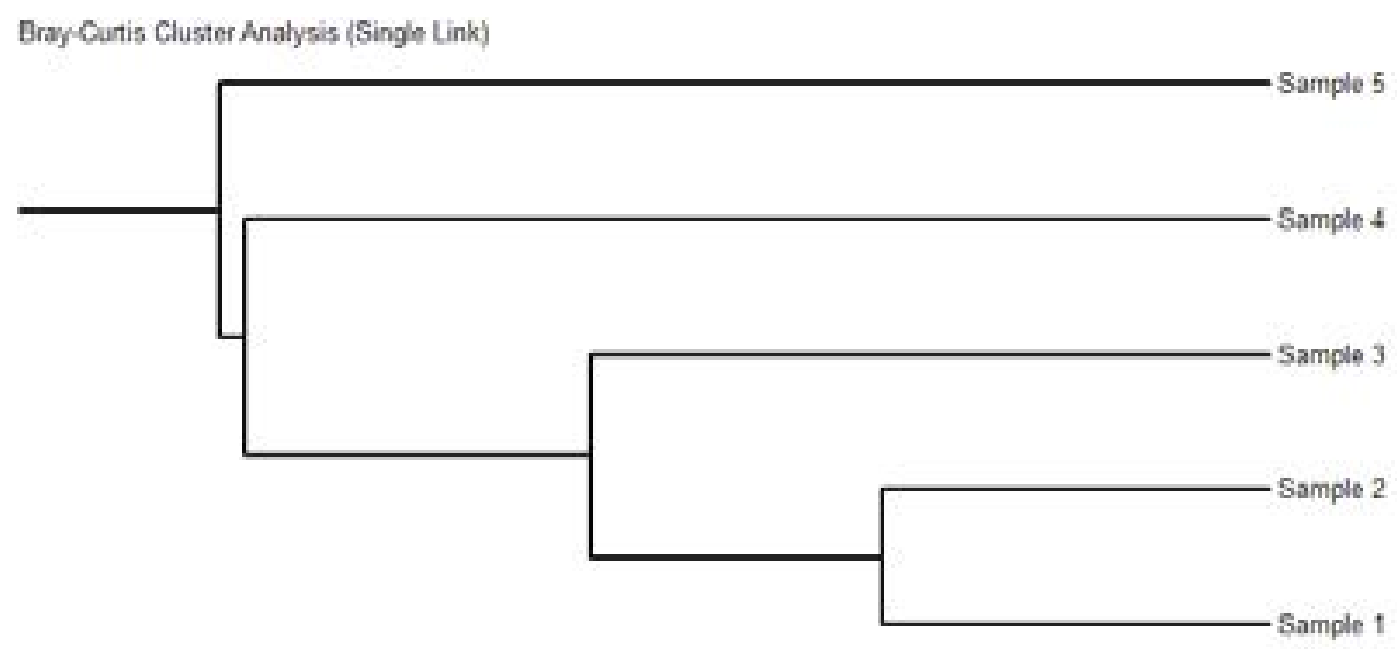

\begin{tabular}{lll}
\hline 0. * Similarity & 50, & 100
\end{tabular}

Figura 4. Dendrograma que muestra la clasificación de las parcelas de un bosque secunario de acuerdo a la composición florística

\section{Diversidad beta}

La similitud florística entre las parcelas de acuerdo a los valores del índice de Jaccard resulto alta con 69\%. El índice de similitud Sorencen mostró una asociación alta de acuerdo a la formación de 4 grupos, donde las parcelas $1,2,3$ y 4 presentan mayor homogeneidad en la estructura florística con una similitud mayor del $68.9 \%$, en la Figura 3 se presenta el dendrograma resultante del análisis de clasificación de las parcelas por su composición florística.

Esto se deriva de la presencia u ocurrencia de estas especies en la mayoría de las muestras, del total de las 5 parcelas, la primera especie se observó en las 5 parcelas, la segunda se registró en la primera y la tercera y la cuarta especie en 3 parcelas. Este parámetro está condicionado por el número de los individuos dentro de la parcela, el patrón espacial y el tamaño de las unidades muestrales. Esta condicionante permite reconocer el grado de uniformidad en la distribución de los individuos de cada una de las especie. Esto quiere decir que aquellas especies que presentan un valor mayor son aquellos que poseen un patrón regular mientras aquellas con valor bajo son características de un patrón agregado irregular y disperso como se observa en el dendrograma de la Figura 4. 
Cuadro 2. Matriz de evaluación del grado de perturbación del bosque secundario del área de estudio. Bajo (B), medio (M) y alto (A)

\begin{tabular}{llllll}
\hline \multicolumn{1}{c}{ Indicadores } & \multicolumn{5}{c}{ Parcela } \\
& $\mathbf{1}$ & $\mathbf{2}$ & $\mathbf{3}$ & $\mathbf{4}$ & $\mathbf{5}$ \\
\hline Área ocupada por caminos & 2 & 5 & 4 & 2 & 4 \\
Grado de extracción de recursos vegetales & 2 & 2 & 2 & 2 & 3 \\
Ocurrencia de fuegos & 3 & 3 & 3 & 3 & 3 \\
Grado de tala & 2 & 2 & 3 & 2 & 2 \\
Actividad de pastoreo & 3 & 3 & 3 & 1 & 2 \\
Incidencia de la vegetación secundaria e introducida & 3 & 2 & 2 & 1 & 2 \\
Grado de perturbación & 2 & 1 & 2 & 2 & 3 \\
Suma de indicadores & 17 & 18 & 19 & 13 & 19 \\
Evaluación del grado de perturbación & $\mathrm{M}$ & $\mathrm{M}$ & $\mathrm{M}$ & $\mathrm{A}$ & $\mathrm{M}$ \\
\hline
\end{tabular}

\section{Grado de perturbación o modificación}

La evaluación de la representatividad de las especies y los estratos originales en la vegetación actual se consideró alta por estar presente especies nativas de estos ecosistemas: en el arbóreo, $S$. parahybum, D. winteri, B. Orellana, B. gasipaes, C. odorata en el arbustivo, $C$. palmata, Megaskepasma erythrochlamys, Cheilocostus speciosus, Calathea lutea y Xanthosoma roseum; y en el herbáceo, Banisteriopsis caapi, Dracaena reflexa, Aphelandra squarrosa, Cordyline fruticose y Axonopus compresus. La cobertura vegetal se evaluó como alta, al obtener que entre el $45-65 \%$ del territorio está ocupado por la vegetación. El grado de modificación o perturbación resultó variable en el área de estudio con calificativo de medio para las consecuencias provocadas por las prácticas extractivistas para la producción de madera y la ampliación de cultivos establecidos en las chakras y la ampliación de las áreas de pastoreo. La puntuación diferenciada por daños ocasionados por la ampliación y ocupación de áreas para los diferentes accesos a la comunidad, debido a su ubicación geográfica con respecto a la trayectoria de estos, todo lo anterior explica los criterios para la calificación otorgada (Cuadro 2). Hobbs y Huenneke (1992), afirman que cualquier cambio en el régimen histórico de disturbio de un ecosistema puede alterar la composición de las especies mediante la disminución de la importancia de las especies nativas, la creación de oportunidades para la introducción de especies exóticas o por las dos vías.

De acuerdo a la valoración realizada en la matriz general de evaluación del grado de perturbación del bosque secundario se obtuvo para cada uno de los criterios área un valor de 15 - 20 que refleja un grado de modificación o perturbación medio en las parcelas $1,2,3$ y 5 , mientras que la parcela 4 con un valor de 5-14 presentado un grado de modificación alto, esto considerando que en el sector donde se ubica esta parcela existe una alteración en el ecosistema debido a la actividad turística.

\section{Conclusiones}

E 1 presente trabajo brindó información cuantitativa Cde la diversidad florística. En las parcelas se identificaron un total de 27951 individuos pertenecientes a 74 especies y a 43 familias. Presentó un predominio de la estructura arbórea con una presencia importante de estratos arbustivos.

Mediante la aplicación de los índices de diversidad y el valor de importancia se reconoció la relevancia de la especie $S$. parahybum. El índice de riqueza específica de Margalef fue de 7.39 y Menhinick fue de 0.44 , lo cual demostró que la vegetación posee una gran 
cantidad de especies. El índice de Simpson fue de 0.87 lo cual demuestra que en el área de estudio no existe una especie dominante, el índice de Shannon- Wiener con valor de 3.01 muestra que la comunidad es equitativa. El índice de Sorensen con 68.9\% y Jaccarad 69\%, lo que permitió establecer que existe una similitud de especies.

El grado de modificación y perturbación del bosque secundario obtuvo un valor de 15 - 20 que refleja un grado de modificación media, en las parcelas 1, 2, 3 y 5 , mientras que la parcela 4 con un valor de 5-14 presentado un grado de modificación alto

\section{Bibliografía}

Álvarez, M., Córdoba, S., Escobar, F., Fagua, G., Gast, F., Mendoza, H., Ospina, M., Umaña, A. M. Y Villareal, H. (2006). Manual de métodos para el desarrollo de inventarios de biodiversidad. Instituto de Investigación de Recursos Biológicos Alexander von Humboldt. Bogotá, Ramos López Editorial.

Cano, E. (2004). Inventario integrado de los recursos naturales de la provincia de la pampa. Clima, Geomorfología, Suelo y Vegetación. Buenos Aires, Instituto Nacional de Tecnología Agropecuaria provincia de La Pampa y Universidad Nacional de La Pampa.

Cerón, MC. (1993). Manual de botánica ecuatoriana, sistemática y métodos de estudio. Ediciones Abya Yala. Quito, ecuador. $315 \mathrm{p}$.

Cottam, G. Y Curtis, J.T. (1956). The use of distance measures in phytosociological sampling. Ecology, 37, 451- 460.

Finol H. (1971). Nuevos parámetros a considerarse en el análisis estructural de las selvas vírgenes tropicales. Revista Forestal Venezolana 21:29-42.

Gentry, H. (1988). Changes in Plant Community Diversity and Floristic composition on Environmental and Geagraphica Gradients. Ann. Missouri Bot. Gard. 75(1):2-34.

Hobbs R.J. y Huenneke L.F. 1992. Disturbance, diversity, and invasion: implications for conservation. Conservation Biology 6:324-337. Hurlbert S.H. 1971.

Koleff P. (2005). Conceptos y medidas de la diversidad beta. En: Halffter G., Soberón J., Koleff P. y Melic A. Eds. Sobre Diversidad Biológica: el Significado de las Diversidades Alfa, Beta y Gamma, pp. 19-40, Monografías 3er Milenio, Zaragoza.

León, Y., Valencia, R., Pitman, N. y Jorgensen, P. M. (Eds.) (2012). Libro rojo de las plantas endémicas del Ecuador 2000. Herbario QCA, Pontificia Universidad Católica del Ecuador, Quito, Ecuador. P. 1-489.

Lot, A. Y F. Chiang. (1986). Manual de herbario (Administración y manejo de colecciones, técnicas de recolección y preparación de ejemplares botánicos). Cons. Nac. de la Flora de México. 1-142. México.

Luebert, F. Y Becerra, P. (1998). Representatividad vegetacional del Sistema Nacional de Áreas Silvestres
Protegidas del Estado (Snaspe) en Chile. Ambiente y Desarrollo, 16 (2), 62 - 69 .

Margalef, R. (1969). El ecosistema pelágico del Mar Caribe. Memoria de la Sociedad de Ciencias Naturales La Salle, $29,5-36$.

Margalef, R. (1995). Ecología. Barcelona, Omega.

Magurran A.E. (1988). Diversidad Ecológica y su Medición. Ediciones Vedrá, Barcelona.

Matos J. Y Ballate D. (2006). ABC de la Restauración Ecológica. Editorial Feijóo, Santa Clara.

Mcaleece N., Gage J.D.G., Lambshead P.J.D. Y Paterson G.L.J. (1997). BioDiversity Professional statistics analysis software. <http://www.sams.ac.uk/research/ software>

Mera, J. (2018). Restauración de espacios degradados en un área de cantera abandonada. Guayaquil: Universidad de Guayaquil.

Patiño, J., Lozano, P., Tipan, C., Navarrete, H., Lopez, R., Asanza, M., Torres, B. (2015). Composición florística y estructura de un bosque siempreverde piemontano de 600 a 700 m.s.n.m. en la cuenca del río Piatúa, Napo, Ecuador. Revista Amazónica Ciencia y Tecnología, 4 (2), 166-192.

Samaniego, E., Garcia, Y., Neill, D., Arteaga, Y., Vargas, J., Rojas, L. (2015). Diviersidad florística de tres sitios de un bosque siempreverde piemontano de la región oriental amazónica del Ecuador. Revista Amazónica Ciencia y Tecnología, 4 (1), 32-47.

Suárez, S. Y Vischi, N. (1997). Caracterización fisonómicoestructural de vegetación serrana (Alpa corral-CórdobaArgentina). Multinequina, 6, 21-32.

Unión Internacional para la Conservación de la Naturaleza (2013). http://www.iucn.org/es/.

Zabalza, M. I., Barreix, J. C. Y Cano, E. (1989). Relevamiento fitosociológico del Parque Nacional Lihué Calel. Revista Facultad Agronomía Universidad Nacional La Pampa, 4 (1), 69-94. 